\title{
CDK2 regulates nuclear envelope protein dynamics and telomere attachment in mouse meiotic prophase
}

\author{
Alberto Viera ${ }^{1}$, Manfred Alsheimer ${ }^{2}$, Rocío Gómez ${ }^{1}$, Inés Berenguer ${ }^{1}$, Sagrario Ortega ${ }^{3}$, Catherine E. Symonds ${ }^{4}$, \\ David Santamaría ${ }^{4}$, Ricardo Benavente ${ }^{2}$ and José A. Suja ${ }^{1, *}$
}

\begin{abstract}
In most organisms, telomeres attach to the nuclear envelope at the onset of meiosis to promote the crucial processes of pairing, recombination and synapsis during prophase I. This attachment of meiotic telomeres is mediated by the specific distribution of several nuclear envelope components that interact with the attachment plates of the synaptonemal complex. We have determined by immunofluorescence and electron microscopy that the ablation of the kinase CDK2 alters the nuclear envelope in mouse spermatocytes, and that the proteins SUN1, KASH5 (also known as CCDC155) and lamin C2 show an abnormal cap-like distribution facing the centrosome. Strikingly, some telomeres are not attached to the nuclear envelope but remain at the nuclear interior where they are associated with SUN1 and with nuclear-envelope-detached vesicles. We also demonstrate that mouse testis CDK2 phosphorylates SUN1 in vitro. We propose that during mammalian prophase I the kinase CDK2 is a key factor governing the structure of the nuclear envelope and the telomere-led chromosome movements essential for homolog pairing.
\end{abstract}

KEY WORDS: Meiosis, CDK2, SUN1, Telomere, Nuclear envelope

\section{INTRODUCTION}

Meiosis represents a specialized type of cell division characterized by the existence of a single round of DNA replication, followed by two rounds of chromosome segregation. In most sexually reproducing organisms, homologous chromosomes must correctly achieve the processes of pairing, recombination and synapsis during the meiotic prophase I in order to prompt the further generation of haploid cells. The tethering of telomeres to the nuclear envelope is considered a prerequisite that facilitates and mediates the initiation and accomplishment of these processes (Koszul and Kleckner, 2009; Kracklauer et al., 2013; Scherthan, 2007; Zickler and Kleckner, 1998). The attachment of telomeres to the nucleoplasmic face of the inner nuclear membrane (INM) of the nuclear envelope occurs at the leptotene stage of meiotic prophase I. These anchoring sites represent nuclear envelope bridges linking the cytoskeleton and the nucleoskeleton that mediate

${ }^{1}$ Unidad de Biología Celular, Departamento de Biología, Facultad de Ciencias, Universidad Autónoma de Madrid, E-28049 Madrid, Spain. ${ }^{2}$ Department of Cell and Developmental Biology, Biocenter, University of Würzburg, D-97074

Würzburg, Germany. ${ }^{3}$ Biotechnology Program, Centro Nacional de

Investigaciones Oncológicas, E-28029 Madrid, Spain. ${ }^{4}$ Molecular Oncology

Program, Centro Nacional de Investigaciones Oncológicas, E-28029 Madrid, Spain.

*Author for correspondence (jose.suja@uam.es)

Received 11 April 2014; Accepted 27 October 2014 the chromosomal movements during prophase I stages (Koszul and Kleckner, 2009; Kracklauer et al., 2013). In animals and fungi, telomeres move on the inner surface of the nuclear envelope and become clustered at a certain nuclear region, adjacent to the centrosome, creating a bouquet-like arrangement of chromosomes during zygotene. Afterwards, telomeres disperse along the nuclear envelope during the pachytene and diplotene stages until their detachment at the transition between diakinesis and prometaphase I.

As shown in recent years, the proper tethering of telomeres to the nuclear envelope is not only mediated by telomeric DNA and the attachment plates of the axial elements and lateral elements of the synaptonemal complex, but by their interactions with other protein components (Alsheimer, 2009). In this regard, the evolutionarily conserved SUN-domain-containing proteins present at the INM are required to tether the telomeres to the nuclear envelope through their interaction with adaptor proteins that mediate their association to the telomeres at the attachment plates (Alsheimer, 2009; Hiraoka and Dernburg, 2009; Koszul and Kleckner, 2009). Moreover, SUN domain proteins interact at the perinuclear space of the nuclear envelope with KASH domain proteins, located at the outer nuclear membrane, to form the socalled LINC complexes that connect the nucleoskeleton and the cytoskeleton (Starr and Fridolfsson, 2010). Thus, SUN domain proteins provide a link to nucleoskeleton structures, such as the nuclear lamina, whereas KASH domain proteins connect to the microtubule or actin-based cytoskeleton. Consistent with this arrangement, it has been recently reported that in mouse meiosis KASH5 associates with SUN1 and SUN2, and with cytoplasmic dynein (Horn et al., 2013; Link et al., 2014; Morimoto et al., 2012). Interestingly, it has also been recently demonstrated that different serine/threonine protein kinases phosphorylate the nucleoplasmic domain of SUN1 at the meiotic nuclear envelope in the nematode Caenorhabditis elegans, and that these modifications are required for telomere movement and attachment to the centrosome (Labella et al., 2011; Penkner et al., 2009; Sato et al., 2009).

Cyclin-dependent kinases (CDKs) are a group of serine/ threonine protein kinases, highly conserved from yeast to humans, which participate in the regulation and progression of the cell cycle and the subsequent cell division. In recent years, however, it has become evident that CDKs demonstrate a high level of functional redundancy. In this context, the only CDK that seems to be essential for the cell cycle is CDK1, the so-called 'mitotic kinase', whose deletion causes cell cycle arrest (Santamaría et al., 2007). In contrast, it has been reported that the previously considered master regulator of cell cycle progression in mammals, namely CDK2, is dispensable for mitotic cell cycle progression and division (Berthet et al., 2003; Ortega et al., 2003; Tetsu and McCormick, 2003). However, a possible role of CDK2 in meiosis arose from the finding that it 
locates at telomeres and at late recombination nodules throughout meiotic prophase I (Ashley et al., 2001). Indeed, the essential role of CDK2 in gametogenesis was demonstrated from the evidence that $C d k 2^{-/-}$mice were infertile, with complete penetrance (Berthet et al., 2003; Ortega et al., 2003). Accordingly, in $\mathrm{Cdk2}^{-/-}$male mice, meiosis arrests in prophase I, triggering the apoptotic pathway in the spermatocytes, which results in the absence of further meiotic stages (Berthet et al., 2003; Ortega et al., 2003). In a previous study, we reported that in male mouse meiosis CDK2 was required for recombination, proper homolog pairing and synapsis, and sex-body formation (Viera et al., 2009). Moreover, we described that in $C d k 2^{-/-}$mutant spermatocytes some telomeres did not attach accurately to the nuclear envelope during prophase I stages, and suggested that this could lead to extensive non-homologous synapsis (Viera et al., 2009).

In the present study, we have performed a detailed analysis of possible modifications in the structure and composition of the nuclear envelope in $C d k 2^{-/-}$spermatocytes. For this purpose, we have investigated the organization of the nuclear envelope as well as the presence, distribution and dynamics of representative nuclear envelope protein components by using immunofluorescence and electron microscopy. Furthermore, we have tested whether mouse testis CDK2 is able to phosphorylate SUN1 in vitro. According to our results, we propose and discuss the putative participation of CDK2 in maintaining the structure and dynamics of the nuclear envelope, as well as its role in driving the attachment of meiotic telomeres to the nuclear envelope and in chromosome movement and bouquet formation, in male mouse meiosis.

\section{RESULTS \\ Cdk2 $^{-l-}$ spermatocytes display an anomalous distribution of SUN1}

We have previously described that in the most advanced meiotic prophase I stage found in $C d k 2^{-/-}$male mice, i.e. in pachytenelike spermatocytes, some telomeres seemed to be in the nuclear interior and thus were not attached to the nuclear envelope (Viera et al., 2009). Given that it has been proposed that SUN domain proteins present at the INM are involved in the anchoring of telomeres to the nuclear envelope in meiocytes from several species (Alsheimer, 2009; Hiraoka and Dernburg, 2009; Koszul and Kleckner, 2009; Kracklauer et al., 2013), we compared the pattern of distribution of SUN1 in wild-type and $C d k 2^{-/-}$ spermatocytes. To this end, we made a double-immunolabeling of SUN1 and SYCP3, a structural protein component of the axial and lateral elements of the synaptonemal complex, to define meiotic staging during prophase I. We used the squashing technique because it preserves the nuclear volume and chromosome condensation, and positioning inside spermatocyte nuclei (Page et al., 1998; Parra et al., 2002). In wild-type leptotene spermatocytes, SUN1 appeared as small spots at the nuclear envelope (Fig. 1A,B), whereas at zygotene most SUN1 spots were present at the ends of axial and lateral elements giving a bouquetlike arrangement (Fig. 1C,D). In pachytene spermatocytes, all SUN1 signals appeared at the ends of synaptonemal complexes, the attachment plates, associated with the nuclear envelope (Fig. 1E,F). Strikingly, in all prophase I $C d k 2^{-/-}$spermatocytes SUN1 was mainly observed as a polarized cap-shaped continuous labeling at the nuclear envelope (Fig. 1G-L). Given that it is known that SUN1 participates in the attachment of the centrosome to the nucleus (Tzur et al., 2006), we studied whether the polarization of SUN1 in mutant spermatocytes was related to the distribution of the centrosome. To address this, we performed a double-immunolabeling of SYCP3 and pericentrin, a component of the pericentriolar material present at the centrosome, and also of pericentrin and SUN1. Our results showed that the centrosome was always located near the nuclei as an unaltered 'cloud' from leptotene up to pachytene both in wild-type and mutant spermatocytes (supplementary material Fig. S1A-J). Interestingly, the SUN1 cap present in all mutant spermatocytes was always facing the centrosome (Fig. 1M,N; supplementary material Fig. $\mathrm{S} 1 \mathrm{~K}-\mathrm{N})$. These results suggest that in the absence of CDK2 the protein SUN1 is still able to become mostly polarized at the INM near the centrosome, as occurs when it associates to telomeres during the bouquet-like arrangement in wild-type zygotene nuclei. However, SUN1 is unable to redistribute along the nuclear envelope in pachytene-like mutant spermatocytes, as occurs in wild-type pachytene spermatocytes when it associates to the attachment plates.

Surprisingly, in addition to the remarkable anomalous and persisting polarization of SUN1 at the nuclear periphery, it also appeared as small foci at the nuclear interior in all prophase I stages in $C d k 2^{-1-}$ individuals (Fig. 1G-L). Therefore, we next analyzed the possible spatial relationship between internal SUN1 foci and internal telomeres. A triple-immunolabeling of SUN1, TRF1, a telomeric protein, and SYCP3 demonstrated that all internal SUN1 foci were associated with internal TRF1 signals (Fig. 1O,P). However, some internal TRF1 signals actually did not colocalize with SUN1 (Fig. 1O,P). This association of SUN1 with internal telomeres denoted an internal localization of the transmembrane protein SUN1, which is remarkable and without precedent in the literature. Given that several SUN1 foci could be detected in a given $C d k 2^{-/-}$spermatocyte, we scored the number of internal SUN1 foci in ten squashed nuclei of each prophase I stage, in two different $C d k 2^{-1-}$ individuals, after the immunolabeling of SUN1 and SYCP3. Our results demonstrated the presence of one up to four internal SUN1 signals in each analyzed spermatocyte (Fig. 2A). In this sense, $50 \%$ of leptotene spermatocytes presented a single SUN1 internal signal, whereas the percentages of spermatocytes with two or three internal SUN1 foci were $30 \%$ and $15 \%$, respectively. Remarkably, only $5 \%$ of leptotene spermatocytes presented four SUN1 foci at their nuclear interior. By contrast, only $20 \%$ of zygotene and pachytene-like spermatocytes displayed only one internal SUN1 signal, whereas four foci were present in other $20 \%$ of spermatocytes. These observations prompted us to analyze the organization of the nuclear periphery in wild-type and $C d k 2^{-/-}$spermatocytes by electron microscopy. In wild-type pachytene spermatocytes, telomeres were attached to the nuclear envelope through the attachment plates, disk-shaped electron-dense plates at the ends of the lateral elements of the synaptonemal complex (Esponda and GiménezMartin, 1972; Liebe et al., 2004) (Fig. 3A). At the attachment sites, both nuclear envelope membranes were thicker and associated with fibrils that bridge the perinuclear space. These fibrils were in apparent continuity with cytoplasmic fibrils that associated with the outer nuclear membrane (Fig. 3A). According to current models, the fibrils in the perinuclear space correspond, at least in part, to SUN proteins, whereas the cytoplasmic fibrils might represent KASH domain proteins (Schmitt et al., 2007). In mutant pachytene-like spermatocytes, we found normally attached telomeres (Fig. 3B). However, other telomeres in the same nucleus did not attach to the INM through attachment plates, but the ends of the lateral elements contacted laterally with the INM (Fig. 3C). In other cases, the ends of the lateral elements contacted with one or two membrane vesicles of $\sim 200 \mathrm{~nm}$ in diameter, that were apparently detaching from the INM (Fig. 3D,E,G). 
WT
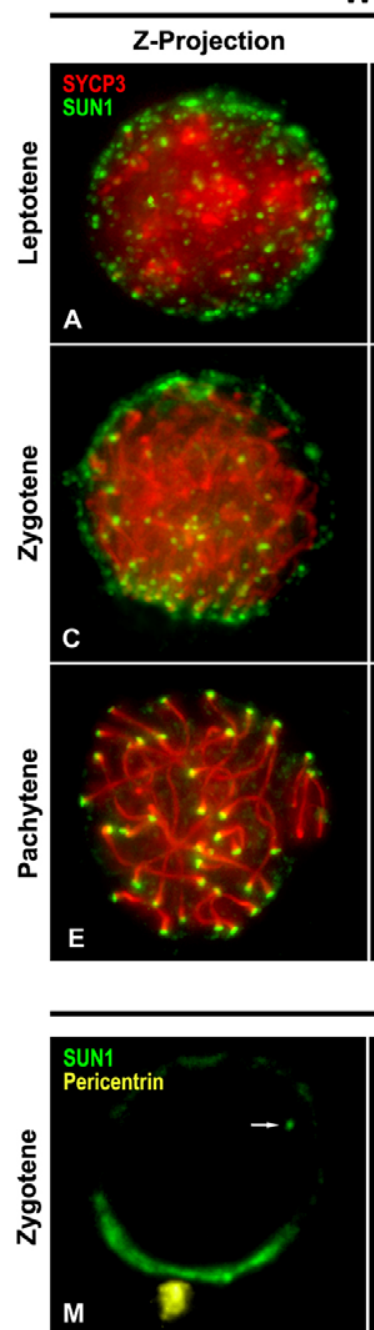

Equator
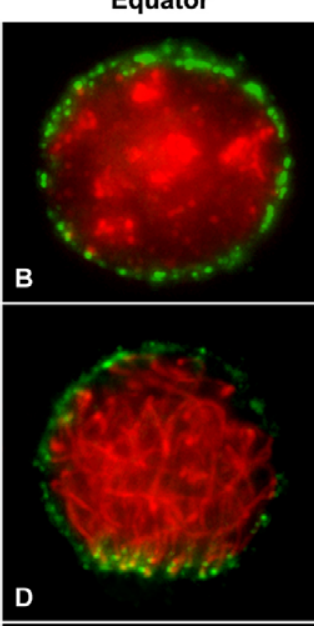

D

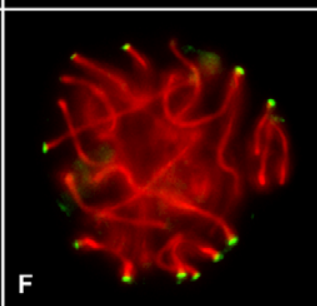

Cdk2 ${ }^{-1-}$
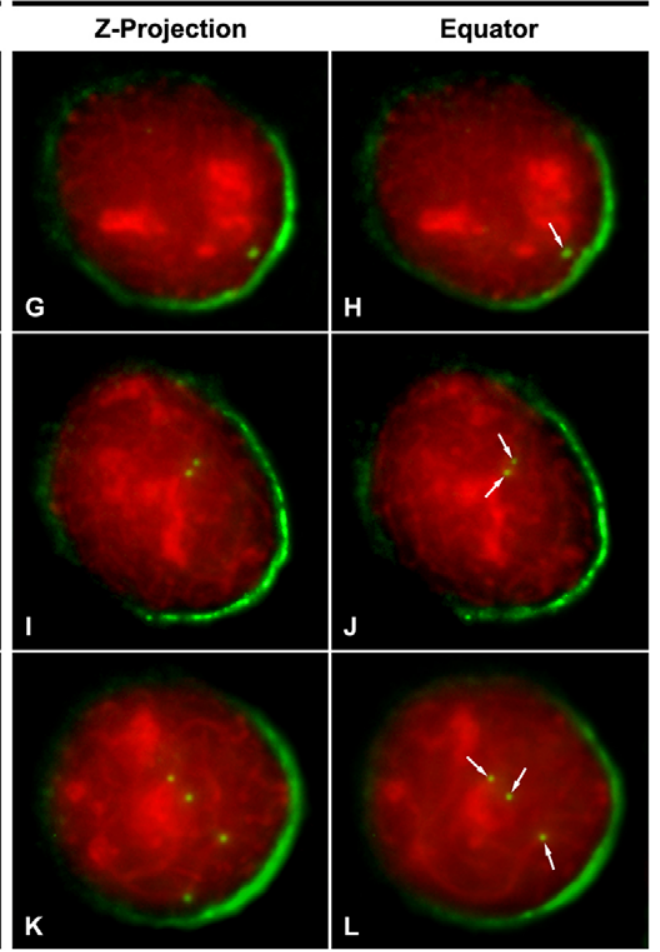

\section{Cdk2 $^{-1}$}

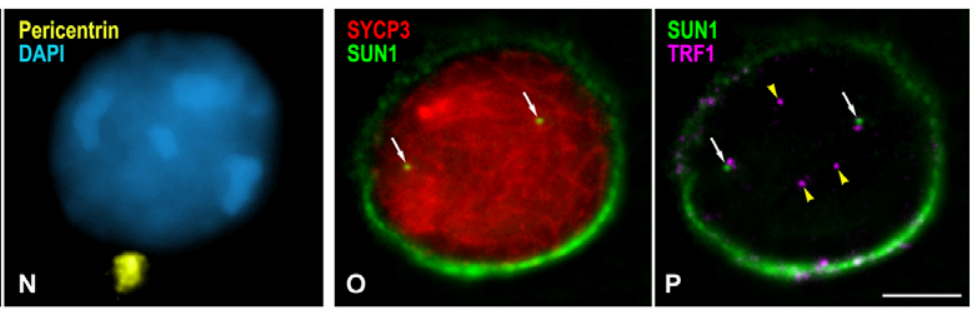

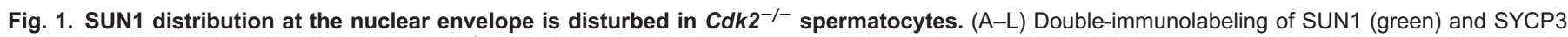
(red) on squashed wild-type (A-F) and Cdk2-/- $(\mathrm{G}-\mathrm{L})$ prophase I spermatocytes. The first and third columns correspond to the $z$-projection of all the focal planes throughout a wild-type and $C d k 2^{-/-}$spermatocyte nucleus, respectively. The second and fourth columns present the partial $z$-projections of 15 focal planes throughout the equatorial region of the same spermatocytes. (A,B) Wild-type (WT) leptotene nucleus. SUN1 appears as spots uniformly distributed at the nuclear periphery. (C,D) WT zygotene nucleus. Most SUN1 spots are polarized at the nuclear envelope reflecting a bouquet-like telomere arrangement.

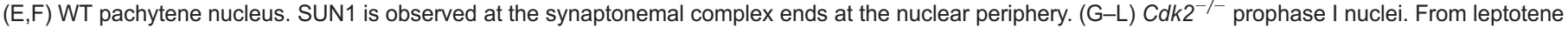
on SUN1 is always found as a continuous and polarized cap-like labeling at the nuclear envelope. Note that SUN1 also appears as small foci (arrows) in the nuclear interior. (M,N) Double-immunolabeling of SUN1 (green) and pericentrin (yellow) on a zygotene Cdk2 ${ }^{-1-}$ spermatocyte. The SUN1 cap faces the pericentrin-labeled centrosome. Note one internal SUN1 spot (arrow). (O,P) Triple-immunolabeling of SUN1 (green), TRF1 (purple) and SYCP3 (red) on a zygotene $\mathrm{Cdk2}^{-/-}$spermatocyte. Both images are partial z-projections of 15 focal planes throughout the equatorial region. Two internal SUN1 foci (white arrows) are near telomeric TRF1 signals, and three internal telomeric signals (yellow arrowheads) do not colocalize with SUN1 spots. Scale bar: $5 \mu$ m.

Interestingly, we also found that the ends of lateral elements or synaptonemal complexes in internal nuclear regions were attached to vesicles that were obviously separated from the nuclear envelope (Fig. 3F,H,I). To our knowledge this is the first description of telomere-associated vesicles in the nuclear interior of meiotic cells.

Taking into account our results, we wondered whether CDK2 could participate in the anchoring of the attachment plates of the synaptonemal complex to the nuclear envelope, taking into account that CDK2 has been cytologically detected at telomeres in mouse prophase I spermatocytes (Ashley et al., 2001). For this purpose, we analyzed by electron microscopy the telomeric location of CDK2 in wild-type pachytene spermatocytes. We found that CDK2 labeling was positioned over the attachment plates that were associated with the INM (Fig. 4A). Given that in C. elegans meiocytes SUN1 is phosphorylated by serine/ threonine protein kinases (Labella et al., 2011; Penkner et al., 2009; Sato et al., 2009), we subsequently tested whether SUN1 might be a substrate for CDK2 phosphorylation in mouse. In order to directly test this possibility we performed an in vitro kinase assay using the N-terminal domain (amino acids 1-243) of SUN1 as a substrate. Interestingly, CDK2 immunoprecipitated from wild-type testis extracts could efficiently phosphorylate recombinant SUN1 (Fig. 4B). Thus, at least in vitro, SUN1 is a substrate for mouse testis CDK2 phosphorylation.

Taken together, our results suggest that CDK2-mediated phosphorylation of SUN1 is involved in its accurate distribution 
A

Leptotene Zygotene Pachytene-like

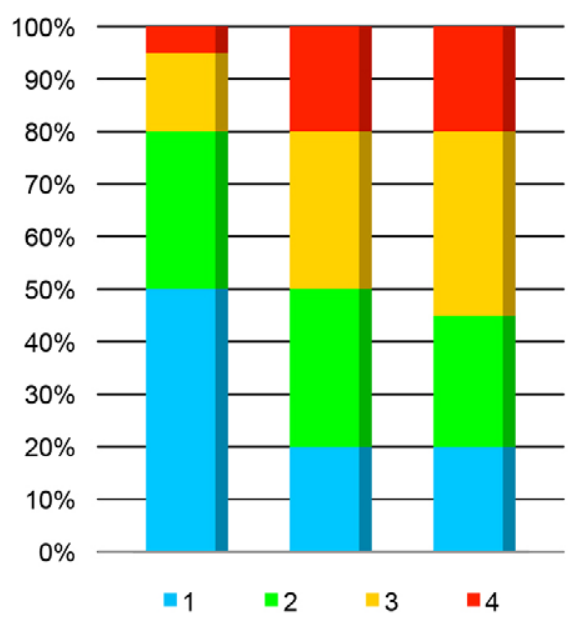

\begin{tabular}{|l|c|c|c|c|}
\hline \multirow{2}{*}{} & \multicolumn{4}{|c|}{ Number of internal SUN1 foci: } \\
\cline { 2 - 5 } & $\mathbf{1}$ & $\mathbf{2}$ & $\mathbf{3}$ & $\mathbf{4}$ \\
\hline Leptotene & $50 \%$ & $30 \%$ & $15 \%$ & $5 \%$ \\
\hline Zygotene & $20 \%$ & $30 \%$ & $30 \%$ & $20 \%$ \\
\hline $\begin{array}{l}\text { Pachytene- } \\
\text { like }\end{array}$ & $20 \%$ & $25 \%$ & $35 \%$ & $20 \%$ \\
\hline
\end{tabular}

B

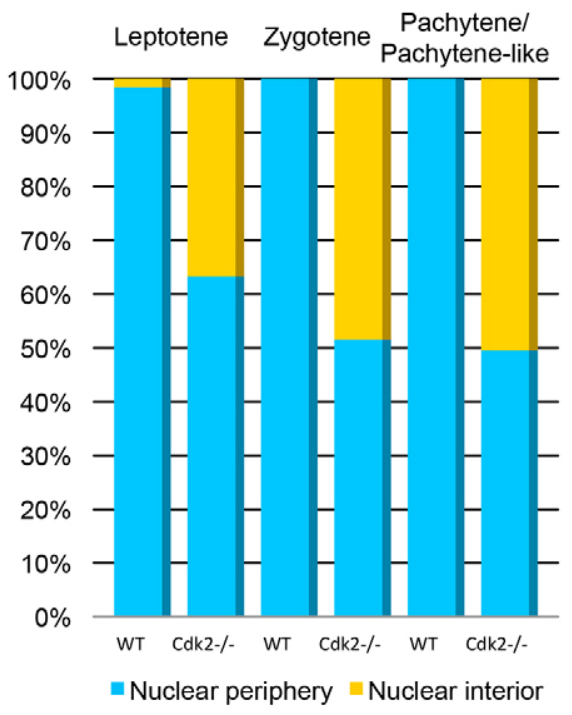

\begin{tabular}{|l|c|c|c|c|}
\hline \multicolumn{5}{|c|}{ Telomere attachment } \\
\hline & \multicolumn{2}{|c|}{ Wild-type } & \multicolumn{2}{c|}{ Cdk2 $^{-/-}$} \\
\cline { 2 - 5 } & $\begin{array}{c}\text { Nuclear } \\
\text { periphery }\end{array}$ & $\begin{array}{c}\text { Nuclear } \\
\text { interior }\end{array}$ & $\begin{array}{c}\text { Nuclear } \\
\text { periphery }\end{array}$ & $\begin{array}{c}\text { Nuclear } \\
\text { interior }\end{array}$ \\
\hline Leptotene & $98.41 \%$ & $1.59 \%$ & $63.28 \%$ & $36.72 \%$ \\
\hline Zygotene & $100 \%$ & $0 \%$ & $51.52 \%$ & $48.48 \%$ \\
\hline $\begin{array}{l}\text { Pachytene/ } \\
\text { Pachytene- } \\
\text { like }\end{array}$ & $100 \%$ & $0 \%$ & $49.48 \%$ & $50.53 \%$ \\
\hline
\end{tabular}

Fig. 2. Percentages of internal SUN1 foci and telomeres.

(A) Percentages of internal SUN1 foci in $\mathrm{Cdk} 2^{-/-}$spermatocytes. The presence of internal SUN1 signals within a nucleus was scored in 20 squashed spermatocytes for each prophase I stage in $\mathrm{Cdk} 2^{-/-}$ individuals. The graphic representation corresponds to the percentage of nuclei showing one (blue), two (green), three (yellow) or four (red) internal SUN1 foci.

(B) Percentages of non-attached telomeres in wild-type and $\mathrm{Cdk} 2^{-/}$ spermatocytes. The position of telomeres within a nucleus was scored in 20 squashed spermatocytes for each prophase I stage in wild-type (WT) and Cdk2 $2^{-/-}$ individuals. The graphic representation corresponds with the percentage of telomeres found at the nuclear periphery (blue) or at the nuclear interior (yellow). and association to the attachment plates found at the ends of axial and lateral elements during prophase I. Thus, in the absence of CDK2 some telomeres are tethered to the nuclear envelope in early prophase I stages, but some of them can detach from the nuclear envelope peeling off vesicles derived from the INM, accounting for the presence of SUN1 at internal telomeres (see below).

\section{KASH5 distribution is disrupted in Cdk2 ${ }^{-1-}$ spermatocytes} In mouse spermatocytes, SUN1 interacts with KASH5 to form LINC complexes associated to the telomeres (Horn et al., 2013; Morimoto et al., 2012). Thus, in wild-type pachytene spermatocytes both SUN1 and KASH5 signals localized at the ends of synaptonemal complexes attached to the nuclear envelope (Fig. 5A-D). Given that the accurate localization of KASH5 during meiosis is disturbed in the absence of SUN1 (Horn et al., 2013; Morimoto et al., 2012), and that we found a cap-shaped polarization of SUN1 in $C d k 2^{-/-}$spermatocytes, we then analyzed the relative distribution of SUN1 and KASH5 in $C d k 2^{-/-}$individuals. $C d k 2^{-/-}$spermatocytes, from leptotene up to the pachytene-like stage, displayed a polarized SUN1 labeling at the nuclear envelope (Fig. 5E,I,M), which was also observed for KASH5 (Fig. 5F,J,N). Indeed, both proteins mostly colocalized at the cap-shaped arrangement (Fig. 5G,K,O). Nevertheless, whereas SUN1 was additionally detected as discrete dots positioned at the nuclear interior, KASH5 was not detectable inside the nuclei (Fig. 5H,L,P). Our observations indicate that CDK2 drives the proper distribution of SUN1 at telomeres in mouse spermatocytes, and that the polarization of SUN1 at the nuclear envelope, in the absence of CDK2, determines the altered distribution of its interacting protein KASH5.

\section{Cdk2 ${ }^{-I-}$ spermatocytes show a disturbed distribution of nuclear envelope components}

Given that we had observed that SUN1, as an INM protein, was polarized at the nuclear envelope in $C d k 2^{-1-}$ spermatocytes, we then explored whether the structure of the nuclear lamina was similarly impaired. To verify this, we performed doubleimmunolabeling experiments of SYCP3 with LAP2, another INM integral protein, or lamin C2, a meiosis-specific isoform of lamin A that appears at the docking sites for meiotic telomeres in the nuclear envelope (Alsheimer and Benavente, 1996; Alsheimer et al., 1999). In wild-type prophase I spermatocytes, LAP2 always appeared to be homogeneously distributed at the nuclear envelope (supplementary material Fig. S2A-F), whereas in $C d k 2^{-1-}$ prophase I spermatocytes we never observed LAP2 labeling at the nuclear envelope (supplementary material Fig. S2G-L). Nevertheless, in $C d k 2^{-1-}$ individuals, LAP2 was able to locate to the nuclear envelope in testicular somatic cells (supplementary material Fig. S2M-P), which are frequently found in squashed preparations of seminiferous tubules. Lamin $\mathrm{C} 2$ showed a rather homogeneous distribution at the nuclear envelope of wild-type spermatocytes during leptotene and zygotene (Fig. 6A-D), but it 


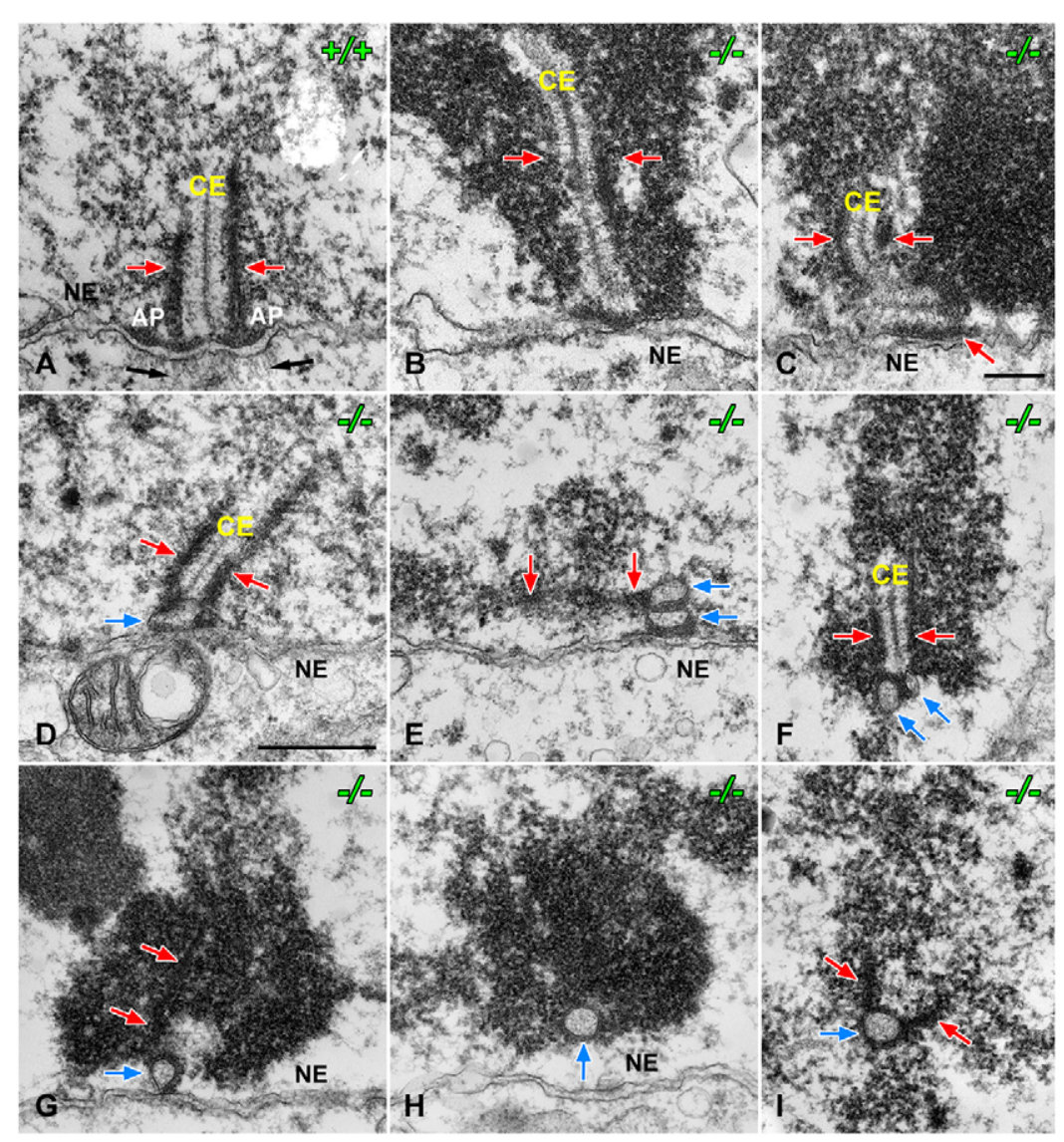

Fig. 3. Internal telomeres are associated with membrane vesicles in Cdk2-/- spermatocytes. (A-I) Electron micrographs showing telomere attachments in wild-type $(+/+)$ pachytene nuclei $(A)$ and in pachytene-like $(-/-)$ mutant nuclei (B-I). (A) End of a synaptonemal complex attached to the nuclear envelope (NE) showing the central element (CE), the two lateral elements (red arrows) and their association to the INM through the attachment plates (AP). Thin fibrils attaching to the cytoplasmic side of the nuclear envelope are indicated (black arrows). (B-I) In mutant spermatocytes some telomere regions appear rather normal (B) whereas in other cases one of the lateral elements (red arrows) interacts laterally with the INM (C). Some other telomere regions appear to be associated with one or two membrane vesicles (blue arrows) either associated to the INM $(D, E, G)$ or lying at the nuclear interior $(F, H, I)$. Scale bars: $200 \mathrm{~nm}(A-C)$; $500 \mathrm{~nm}(\mathrm{D}-\mathrm{I})$. showed a patchy distribution in pachytene (Fig. 6E,F; see also Alsheimer et al., 1999). Surprisingly, in all $C d k 2^{-/-}$prophase I spermatocytes lamin C2 appeared to be polarized showing a capshaped labeling at the nuclear envelope (Fig. 6G-L). Given that this labeling is similar to that found for SUN1 in mutant spermatocytes, we co-detected lamin $\mathrm{C} 2$ and SUN1 to assess whether these proteins colocalized at the nuclear envelope cap. Our observations demonstrated that indeed both proteins colocalized at the aberrant nuclear envelope cap in mutant spermatocytes (Fig. 6M-P). Interestingly, some lamin $\mathrm{C} 2$ foci were detected at the nuclear interior, however, these foci never colocalized with internal SUN1 foci (Fig. 6N-P). These results demonstrate that, in the absence of CDK2, the distribution of SUN1 at the INM and the structure of the nuclear lamina are severely disturbed, with a complete lack of LAP2.

Taking into account the drastic alterations in the distribution of the nuclear envelope proteins SUN1, KASH5, LAP2 and lamin $\mathrm{C} 2$ in $C d \mathrm{k}^{-/-}$spermatocytes, we also explored whether the distribution of nuclear pore complexes (NPCs) was also affected. For this purpose we performed a double-immunolabeling of SYCP3 and RanGAP1, a Ran GTPase-activating protein that localizes at the cytoplasmic filaments of NPCs (Mahajan et al., 1997), in both wild-type and $C d k 2^{-/-}$prophase I spermatocytes. We observed that RanGAP1 was distributed as discontinuous patches along the nuclear envelope in wild-type prophase I spermatocytes. Accordingly, the projections of equatorial focal planes revealed a discontinuous ring surrounding the nuclei of these spermatocytes (supplementary material Fig. S3A-F). Likewise, a homogeneous patchy distribution of NPCs was observed in $C d k 2^{-/-}$prophase I spermatocytes (supplementary material Fig. S3G-L). Thus, the absence of CDK2 does not affect the distribution of NPCs.

\section{Telomere tethering to the nuclear envelope is altered in Cdk2 ${ }^{-I-}$ spermatocytes}

The abnormal cap-shaped distribution of several nuclear envelope components in $\mathrm{Cdk2}^{-/}$spermatocytes prompted us to investigate telomere dynamics at the nuclear envelope in these individuals. For this purpose, we determined the distribution and behavior of telomeres in both wild-type and $C d \mathrm{k}^{-/-}$ spermatocytes during early prophase I stages by a doubleimmunolabeling of the telomeric protein TRF1 and SYCP3 (Fig. 7). In wild-type spermatocytes, telomeric TRF1 signals were discernible at the ends of the developing axial elements, as revealed by the anti-SYCP3 antibody during leptotene (Fig. 7A). The $z$-projections of all focal planes throughout these nuclei showed a homogeneous nuclear distribution of telomeres. Progressively, most telomere signals were polarized at a discrete nuclear region during zygotene denoting a bouquet-like arrangement (Fig. 7C). In pachytene spermatocytes, telomeres redistributed along the nuclear envelope and the bouquet-like arrangement was disorganized (Fig. 7E). In $C d k 2^{-1-}$ spermatocytes, TRF1 signals appeared to be dispersed at the nuclear envelope and they never presented a polarized distribution (Fig. 7G,I,K), and thus a bouquet-like arrangement was never observed in zygotene nuclei (Fig. 7I). This observation corroborates our previous proposal that in $C d \mathrm{k}^{-/-}$spermatocytes the presence of telomeres that did not attach to the nuclear envelope might 

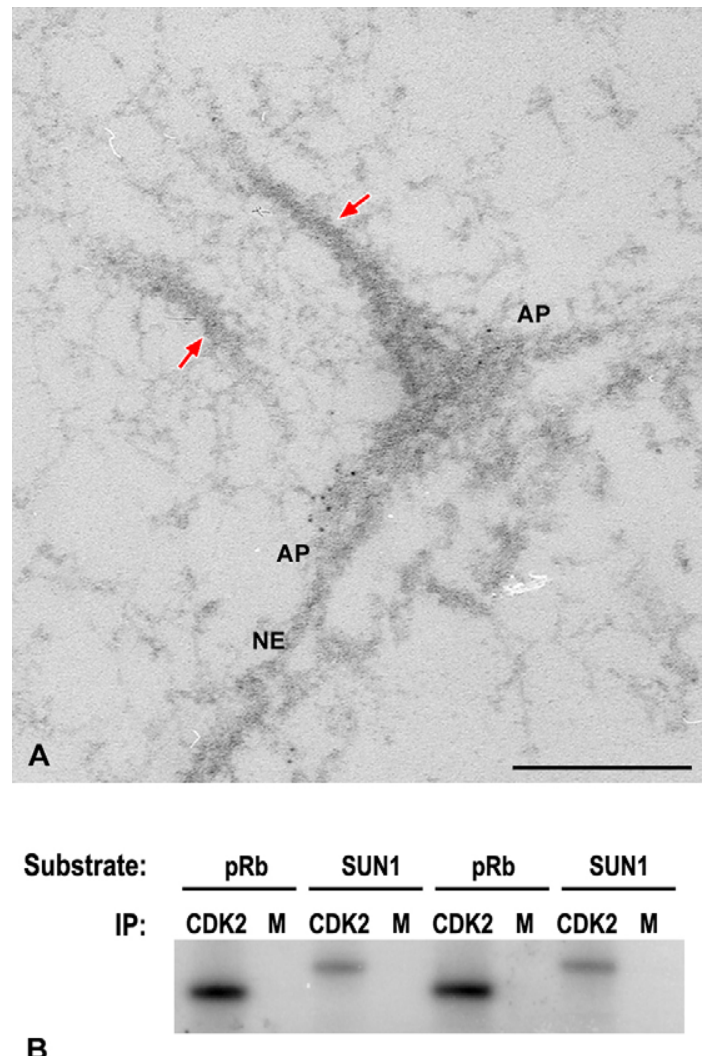

Fig. 4. CDK2 locates at the attachment plates and phosphorylates SUN1 in vitro. (A) Immunogold EM examination of CDK2 in wild-type (+/+) mouse pachytene spermatocytes. CDK2 locates at the attachment plates (AP) of the lateral elements (red arrows) that are connected with the INM of the nuclear envelope (NE). Scale bar: $200 \mathrm{~nm}$. (B) In vitro kinase assay. Kinase activity associated with CDK2 immunoprecipitates obtained from 8week-old wild-type testis extracts. Recombinant SUN1 (amino acids 1-243) together with the canonical CDK substrate pRb (amino acids 773-928) were used in parallel. Results from two independent experiments are shown. M, mock immunoprecipitate.

prompt the absence of bouquet formation, which ultimately leads to the extensive non-homologous pairing found (Viera et al., 2009).

Taking into account our previous observation of internal telomeres in $C d \mathrm{k}^{-/-}$spermatocytes (Viera et al., 2009) and the presence of SUN1 signals at internal telomeres associated with membrane vesicles, we next investigated the origin of the failures in telomere attachment to the nuclear envelope. To determine whether the internal telomeres found in $C d k 2^{-1-}$ pachytene-like spermatocytes arise by either their detachment from the nuclear envelope, tearing SUN1-containing membrane vesicles, or by their inability to attach to the nuclear envelope during preceding meiotic stages, we analyzed early prophase I stages in wild-type and $C d k 2^{-1-}$ spermatocytes. To address this, we estimated the location of telomeres (either peripheral or internal) in ten nuclei of each prophase I stage in two different individuals for each genotype (wild-type and $C d k 2^{-/-}$) after the immunostaining of TRF1 and SYCP3. The analyses of partial projections corresponding to the equatorial regions of wild-type prophase I spermatocytes demonstrated that $1.59 \%$ of the TRF1 telomere signals were present at the nuclear interior in leptotene nuclei (Fig. 7A,B; Fig. 2B). These internal telomeric signals were no longer observed in wild-type zygotene and pachytene nuclei
(Fig. 7C-F; Fig. 2B). By contrast, in $C d k 2^{-/-}$spermatocytes a high percentage of internal telomeric signals were always observed in leptotene (36.72\%), zygotene (48.48\%) and pachytene-like $(50.52 \%)$ spermatocyte nuclei, respectively (Fig. 7G-L; Fig. 2B).

We also wondered whether the internal TRF1 signals that we observed in $C d k 2^{-/-}$spermatocytes corresponded to proximal telomeres (located at the chromosome centromeric end) or distal telomeres (located at the non-centromeric end), given that mouse chromosomes are telocentric. To examine this, we performed both a double-immunolabeling of centromeres and SYCP3 (Fig. 8), and a triple detection of telomeres, centromeres, and SYCP3 (supplementary material Fig. S4). Our results showed that in wild-type spermatocytes the centromeres were located at the nuclear periphery from leptotene up to pachytene (Fig. 8AF; supplementary material Fig. S4A-D). However, in $C d k 2^{-/-}$ spermatocytes the centromere signals were found, not only at the nuclear periphery, but also at the nuclear interior in all prophase I stages (Fig. 8G-L; supplementary material Fig. S4E-P). The simultaneous labeling of centromeres and telomeres showed that in mutant spermatocytes both the proximal telomeres, with TRF1 and ACA signals, and the distal telomeres, only with TRF1 signals, were likewise present at the nuclear interior at all prophase I stages (supplementary material Fig. S4E-P). Taken together, our results indicate that in the absence of CDK2 some telomeres, independently of their proximal or distal chromosomal position, are unable to attach to the nuclear envelope during leptotene and remain at the nuclear interior in later prophase I stages, and a bouquet-like arrangement is not reached. Moreover, the presence of intranuclear telomeres associated with nuclear-envelope-derived membrane vesicles (Fig. 3D-I) and with SUN1 (Fig. 1P) indicates that some attached telomeres have detached from the nuclear envelope in the course of meiotic prophase I.

\section{DISCUSSION CDK2 regulates SUN1 distribution at the meiotic nuclear envelope}

SUN1 is a member of the SUN domain protein family that is known to participate in the attachment of meiotic telomeres to the INM of the nuclear envelope (Alsheimer, 2009; Hiraoka and Dernburg, 2009; Koszul and Kleckner, 2009; Kracklauer et al., 2013). In wild-type mice, SUN1 is enriched at discrete nuclear envelope docking sites that physically connect the telomeres of meiotic chromosomes to the nuclear envelope (Ding et al., 2007). By contrast, we found that in the $C d k 2^{-/-}$background most SUN1 arranges into a cap-like structure polarized at the nuclear envelope region facing the centrosome. This result therefore indicates that CDK2 is not essential for the association of SUN1 to the INM. Interestingly, we have additionally observed that the SUN1 cap at the nuclear envelope is detected in all prophase I stages in these mutant spermatocytes. This finding suggests that SUN1 is able to load to the INM, but that it cannot disperse to discrete regions in the absence of CDK2. Accordingly, we suggest that during mammalian meiosis CDK2 regulates the lateral diffusion of SUN1 along the nuclear envelope. Our data demonstrate at the ultrastructural level that CDK2 locates at the attachment plates of the synaptonemal complex, where SUN1 participates in telomere anchoring to the nuclear envelope (Ding et al., 2007), and that mouse testis CDK2 phosphorylates SUN1 in vitro. Thus, the cytological and biochemical data together indicate that the phosphorylation of the nucleoplasmic domain of 


\section{WT}
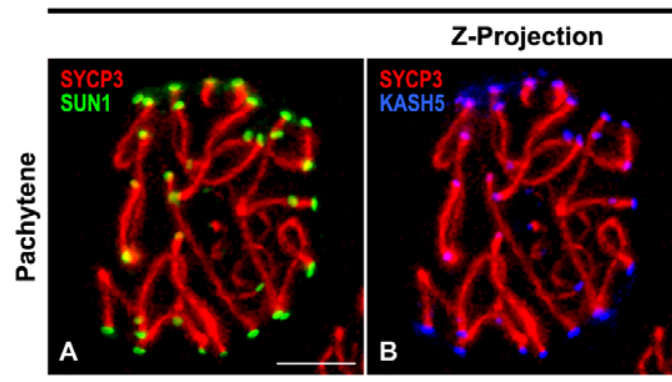

Cdk2-
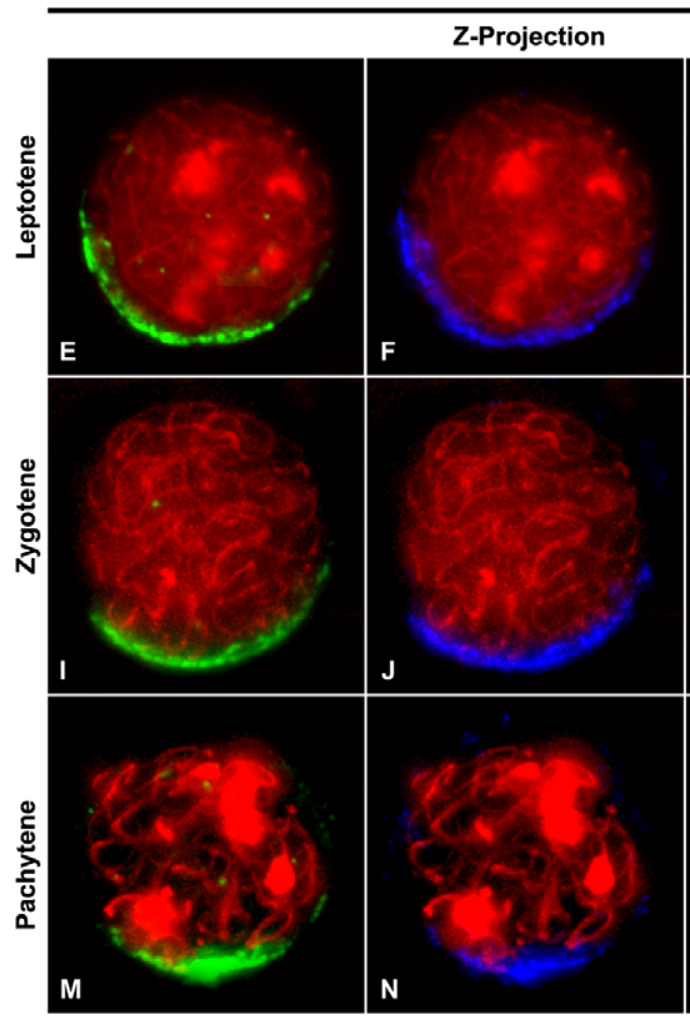
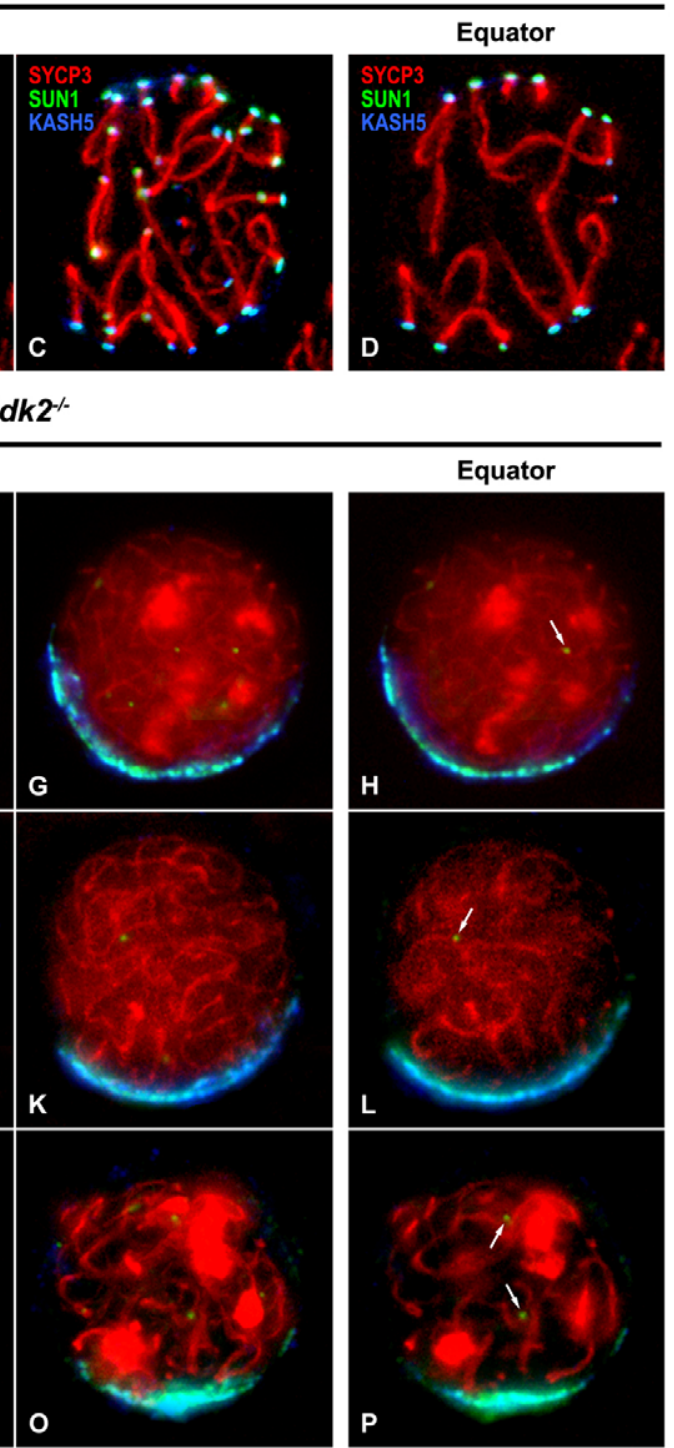

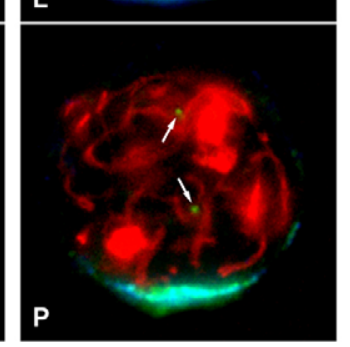

Fig. 5. SUN1 and KASH distribution in $\mathrm{Cdk2}^{-/-}$ spermatocytes. Triple-

immunolabeling of SUN1 (green), KASH5 (blue) and SYCP3 (red) on pachytene wild-type (WT) (A-D) and

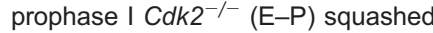
spermatocytes. The first three columns correspond to the $z-$ projection of all the focal planes throughout a nucleus. The fourth column presents the partial $z-$ projections of 15 focal planes throughout the equatorial region of the same nuclei. (A-D) WT pachytene nucleus. SUN1 and $\mathrm{KASH} 5$ are positioned at discrete sites of the nuclear envelope, coincident with the attachment plates of the synaptonemal complex, and thus they are detected at the nuclear periphery. (E-P) $C d k 2^{-/-}$prophase I spermatocytes. In all prophase I stages, SUN1 and KASH5 are invariably distributed as a continuous and polarized cap-like arrangement at the nuclear envelope. Although SUN1 also appears as small foci (arrows) at the nuclear interior, $\mathrm{KASH} 5$ is not detectable at these foci. Scale bar: $5 \mu \mathrm{m}$.
SUN1 by CDK2 could be an important step in regulating SUN1 distribution along the nuclear envelope in spermatocytes. Interestingly, two recent studies have reported that the kinases CHK-2 and PLK-2, by phosphorylating the nucleoplasmic domain of SUN-1 during C. elegans meiosis, do not regulate telomere attachment, but the telomere movement along the nuclear envelope and homolog pairing (Labella et al., 2011; Penkner et al., 2009; Sato et al., 2009). Similarly, in mouse meiosis CDK2 could be responsible for phosphorylating SUN1 to direct the lateral diffusion of these LINC complexes and then move telomeres along the nuclear envelope. Accordingly, in $C d k 2^{-/-}$individuals the KASH domain protein that interacts with SUN1 to form meiotic LINC complexes, namely KASH5, presents an altered distribution, which is coincident with SUN1 at the nuclear envelope. Thus, our data indicate that CDK2 is dispensable for the interaction of SUN1 and KASH5 at LINC complexes, and support recent reports stating that in male mouse meiosis the localization of KASH5 is to some extent dependent on SUN1 (Horn et al., 2013; Link et al., 2014; Morimoto et al., 2012). Indeed, it has been demonstrated that in mouse meiosis SUN1-KASH5 complexes are necessary to promote chromosome pairing, and the proper progression of prophase I, by generating a nucleocytoplasmic bridge connecting telomeres to microtubules (Horn et al., 2013; Link et al., 2014; Morimoto et al., 2012). Consequently, the defective distribution of LINC complexes along the nuclear envelope of $C d k 2^{-1-}$ spermatocytes results in a lack of telomere and chromosome movements, which would promote failures in homologue pairing, accounting for the defective synapsis previously reported in these individuals (Viera et al., 2009). Therefore, we propose that CDK2 is needed to transfer the microtubule-dependent cytoplasmic forces to move telomeres along the meiotic nuclear envelope.

\section{CDK2 regulates the structure of the prophase I nuclear envelope}

Our results show that in the absence of CDK2 the laminassociated protein LAP2 is not detected at the nuclear envelope during prophase I. By contrast, in $C d k 2^{-/-}$mutants LAP2 is still detected at the nuclear envelope of somatic testicular cells, as in rat testis (Schmitt et al., 2007). These results thus suggest that CDK2 might be involved in the regulatory mechanism that allows the association of LAP2 to the INM during meiosis. 
WT
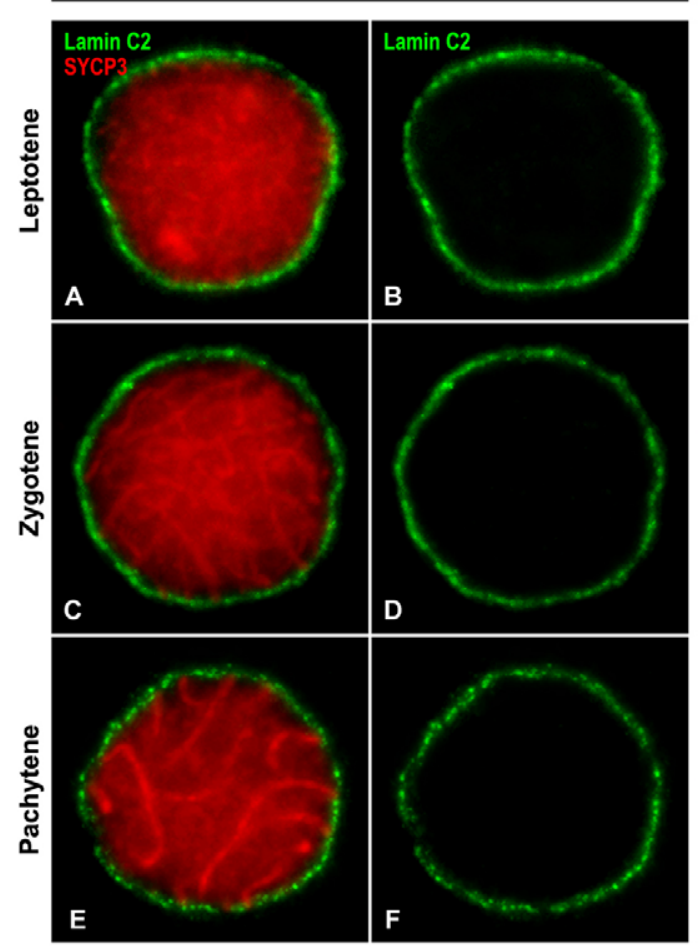
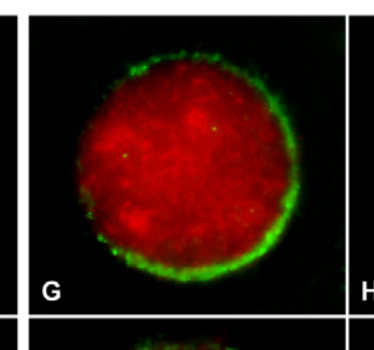

Cdk2-

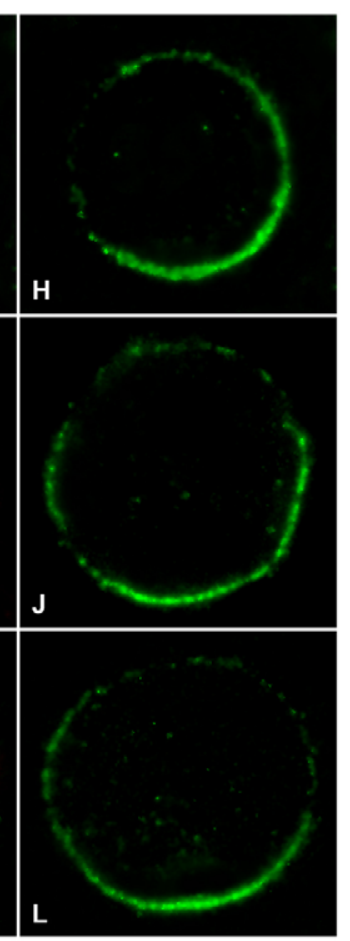

Cdk2
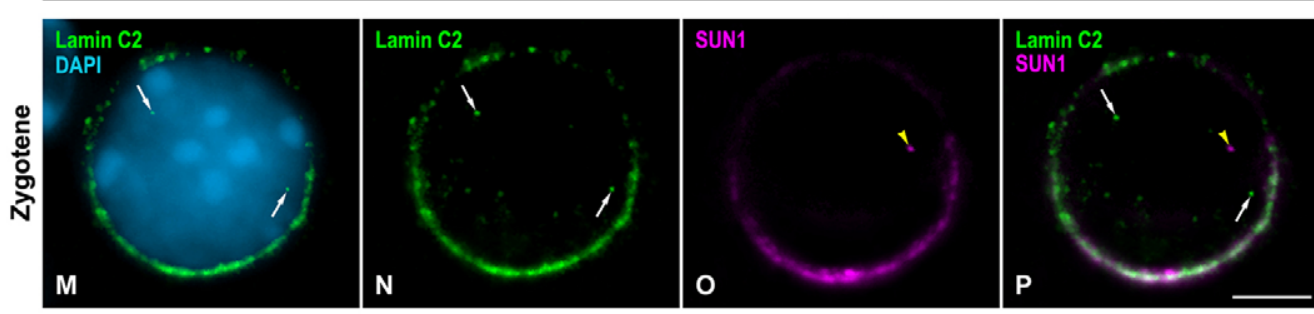

Fig. 6. Lamin $\mathrm{C} 2$ distribution is disturbed in $\mathrm{Cdk2}^{-/-}$

spermatocytes. (A-L) Double-

immunolabeling of lamin C2 (green) and SYCP3 (red) on squashed wildtype (WT) (A-F) and Cdk2 $2^{-/-}$

$(\mathrm{G}-\mathrm{L})$ prophase I spermatocytes. The images correspond to the partial $z$ projections of 15 focal planes throughout the equatorial region of each spermatocyte. (A-F) WT prophase I nuclei. Lamin $\mathrm{C} 2$ is uniformly distributed at the nuclear envelope. (G-L) Cdk2 $2^{-/-}$prophase I nuclei. Lamin C2 appears to be polarized, showing a cap-shaped labeling at the nuclear envelope. (M-P) Equatorial z-projection of a squashed $C d k 2^{-/-}$zygotene nucleus double-immunolabelled for lamin C2 (green) and SUN1 (purple) and chromatin counterstaining with DAPI (blue). Lamin C2 and SUN1 colocalize showing a cap-like distribution at the nuclear envelope. Some internal lamin C2 spots (white arrows) are observed but they do not colocalize with SUN1 internal focus (yellow arrowhead). Scale bar: $5 \mu \mathrm{m}$.

Consequently, it should be addressed in further investigations whether CDK2 participates in the regulation of the phosphorylation pathway of LAP2 during mammalian meiosis.

We have also found that in $C d k 2^{-1-}$ spermatocytes the distribution of lamin $\mathrm{C} 2$ at the nuclear envelope is disturbed. Indeed, lamin C2 unusually shows a cap-like distribution facing the centrosome. Given that in mammals the meiotic A-type lamin, lamin C2, is exclusively expressed during prophase I (Alsheimer and Benavente, 1996; Furukawa et al., 1994; Smith and Benavente, 1992), it can be assumed that CDK2 is involved in the assembly of the meiosis-specific nuclear lamina. In the $C d k 2^{-/-}$background, the distribution of lamin C2 at the nuclear envelope during meiosis is surprisingly coincident with that observed for SUN1. SUN1 and its paralog SUN2 are present at the INM of the nuclear envelope in both somatic and germ cells, and they are able to interact with A-type lamins directly through their nucleoplasmic domain (Alsheimer, 2009; Gerace and Huber, 2012; Tzur et al., 2006). During mouse meiosis, SUN1, SUN2 and lamin C2 appear to be enriched at the attachment sites of telomeres to the nuclear envelope (Alsheimer et al., 1999; Alsheimer et al., 2000; Ding et al., 2007; Schmitt et al., 2007). Hypothetically, two possible pathways can determine the altered distribution of SUN1 and lamin $\mathrm{C} 2$ in $C d \mathrm{k}^{-/-}$spermatocytes. One possibility is that CDK2 regulates the localization of SUN1 and lamin $\mathrm{C} 2$ in an independent manner, and that its ablation alters the distribution of both proteins individually. Alternatively, CDK2 might participate in a pathway that regulates only one of these proteins, either SUN1 or lamin C2, one of which determines the dynamics of the other. In this sense, it has been recently demonstrated, in somatic cells, that the interaction of SUN1 with lamin A is disrupted after the phosphorylation of SUN1 by CDK1 or PLK1 (Patel et al., 2014). However, previous reports have demonstrated that mutant mice lacking SUN1 do not present an altered organization of the lamin-B-based nuclear lamina during meiosis (Ding et al., 2007), and conversely, the absence of lamin C2 does not promote a modification of SUN1 or SUN2 distribution at telomeres (Link et al., 2013; Schmitt et al., 2007). Indeed, despite the fact that it has been demonstrated that in somatic cells SUN1 presents an extremely strong interaction with A-type lamins, SUN1 localization at the nuclear envelope is independent of lamin A (Crisp et al., 2006). Moreover, in Cdk2 $2^{-1-}$ spermatocytes all the internal SUN1 foci were associated with internal telomeres, whereas the internal lamin C2 signals were never located at internal telomeres or close to internal SUN1 foci. Taking into account our data, and previous results, it can be assumed that the localization of SUN1 and lamin C2 at meiotic telomere attachment sites is independent from each other. However, we suggest that CDK2-mediated phosphorylation 
WT

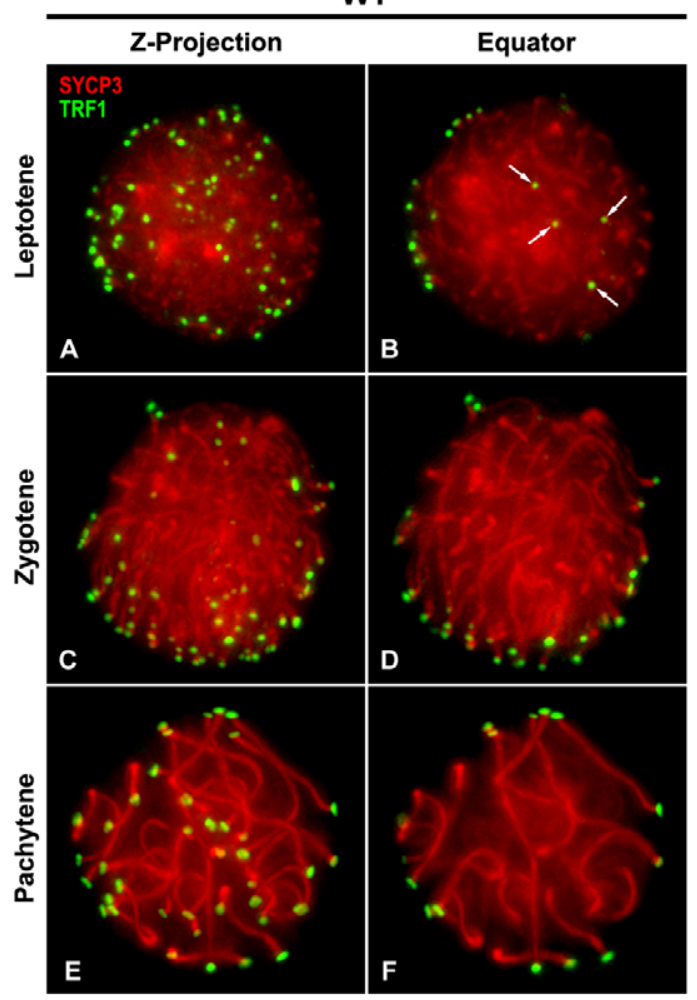

Cdk2-

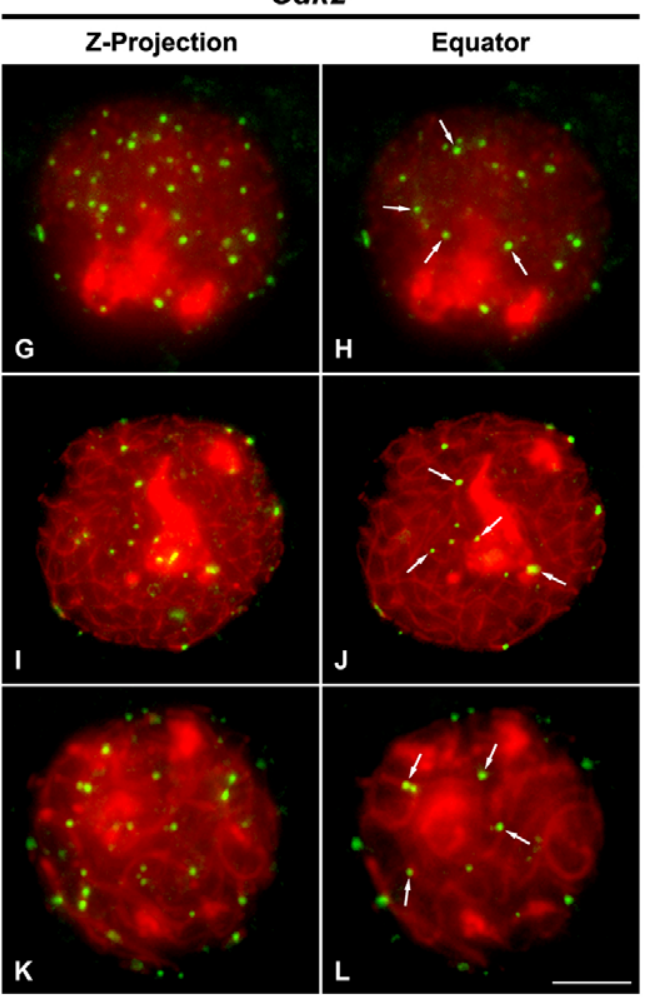

Fig. 7. Telomeres do not attach accurately to the nuclear envelope in $\mathrm{Cdk2}^{-/}$spermatocytes. Doubleimmunolabeling of TRF1 (green) and SYCP3 (red) on squashed wild-type (WT) (A-F) and Cdk2 $2^{-1}$

(G-L) prophase I spermatocytes. The first and third columns correspond to $z$-projections of all focal planes throughout a nucleus. The second and fourth columns show the partial $z$-projections of 15 focal planes throughout the equatorial region of the same nuclei. $(A, B)$ WT leptotene nucleus. Some telomeric TRF1 signals (arrows) are evident at the nuclear interior. (C-F) WT zygotene and pachytene nuclei. No internal TRF1 signals are observed. Note the bouquet-like arrangement of most telomeres associated to the nuclear envelope in the zygotene nucleus, and the telomere signals at the synaptonemal complex ends in pachytene. (G-L) $C d k 2^{-/-}$prophase I nuclei. Internal TRF1 signals

(arrows) are evident in all prophase I nuclei. The bouquet-like arrangement of telomeres is not observed in zygotene nuclei. Scale bar: $5 \mu \mathrm{m}$.
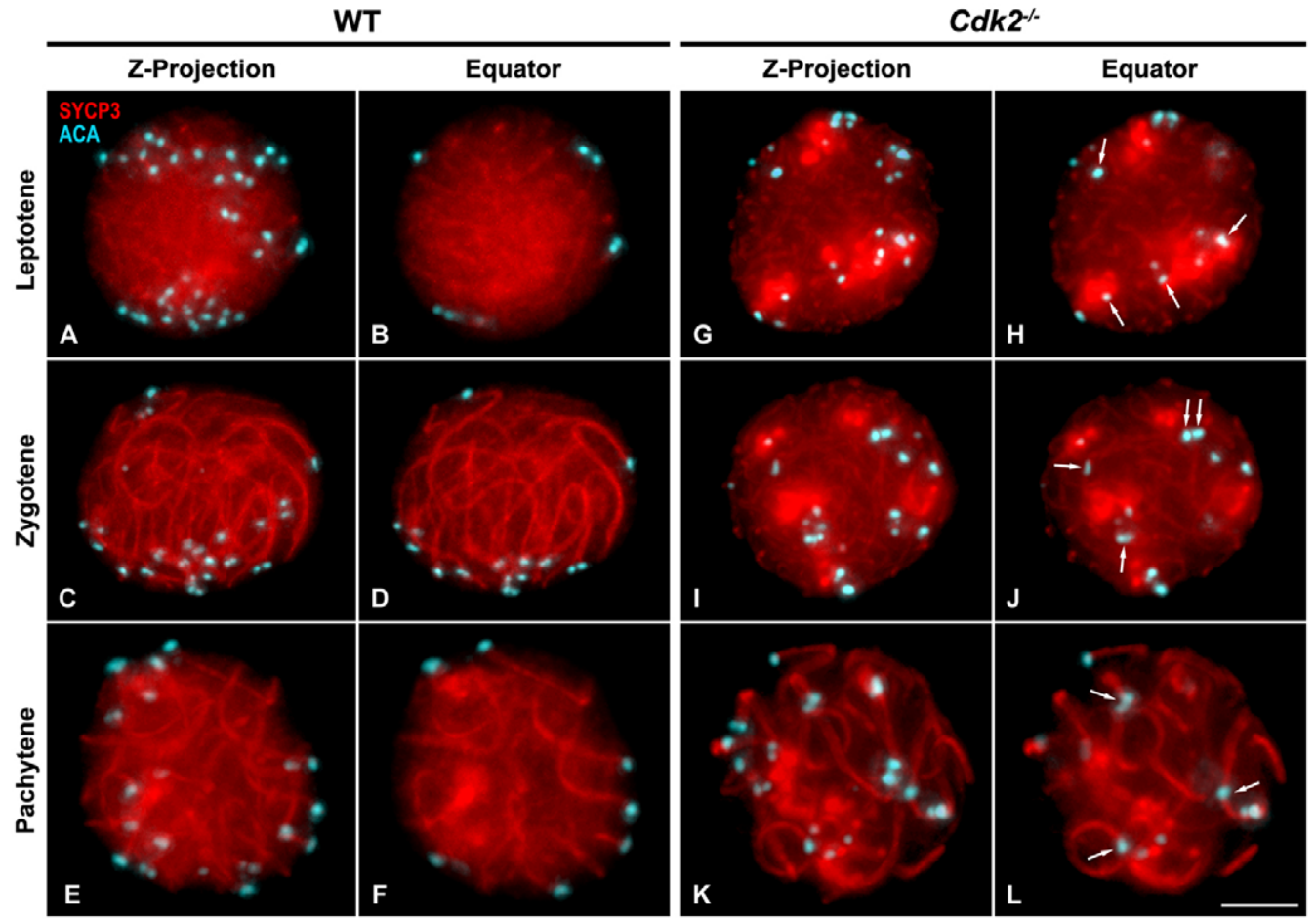

Fig. 8. Centromere distribution is disturbed in $\mathrm{Cdk2}^{-/-}$spermatocytes. Double-immunolabeling of centromeres (ACA, blue) and SYCP3 (red) on squashed wild-type (WT) (A-F) and Cdk2 $2^{-1-}(\mathrm{G}-\mathrm{L})$ prophase I spermatocytes. The first and third columns correspond to the $z$-projection of all the focal planes throughout a WT and Cdk2 $2^{-1-}$ spermatocyte, respectively. The second and fourth columns present the partial z-projections of 15 focal planes throughout the equatorial region of the same nuclei. (A-F) WT leptotene, zygotene and pachytene nuclei. Centromere signals at one of the ends of axial and lateral elements are located at the nuclear periphery from leptotene up to pachytene. Note the bouquet-like arrangement of centromeres, indirectly labeling the proximal chromosome ends, in the zygotene nucleus. (G-L) $\mathrm{Cdk}^{-/}$prophase I nuclei. Some centromere signals (arrows), representing proximal telomere ends, lie at the nuclear interior from leptotene up to the pachytene-like stage. Scale bar: $5 \mu \mathrm{m}$. 
might specifically promote the positioning of lamin $\mathrm{C} 2$ at the nuclear envelope, and/or its redistribution to the telomere-docking sites, as occurs for SUN1. The bulk of our results therefore demonstrate that the ablation of CDK2 causes a severe disruption in the structure of the meiotic nuclear envelope in mouse spermatocytes. Thus, we propose CDK2 as an essential kinase, the first one described in mammals, which regulates the assembly and dynamics of the nuclear envelope during meiotic prophase I.

\section{CDK2 mediates meiotic telomere attachment and dynamics}

Meiotic telomere attachment to the nuclear envelope and their further clustering at a discrete region, which ensures the bouquetlike arrangement of chromosomes, represents a shared feature among a variety of species to facilitate the proper achievement of homology search, recombination and synapsis (Koszul and Kleckner, 2009; Scherthan, 2007; Zickler and Kleckner, 1998). In this sense, our data indicate that the absence of CDK2 inhibits the formation of the bouquet-like arrangement in spermatocytes, which could account for the defects in the processes of homolog pairing, recombination and synapsis previously reported (Viera et al., 2009). Surprisingly, in fifth and sixth generation telomerase-deficient mice $\left(\operatorname{Terc}^{-1-}\right)$, in which telomeric DNA repeats shortened down to below detection levels (Franco et al., 2002), and in shelterin protein Rapl-knockout mice (Scherthan et al., 2011), telomeres are still able to properly attach to the nuclear envelope. In striking contrast, in mice defective for SUN1, telomeres are frequently found at the nuclear interior during prophase I, revealing the importance of non-telomeric proteins for meiotic telomere attachment (Ding et al., 2007; Link et al., 2014). Given that CDK2 locates at late recombination nodules and at telomeres during mouse prophase I (Ashley et al., 2001; Viera et al., 2009), it has been proposed that CDK2 participates in the organization of the Holliday junction intermediates and telomeric DNA t-loops, which share structural similarities (Ashley et al., 2001). Indeed, in $C d k 2^{-/-}$spermatocytes late recombination nodules were never found (Viera et al., 2009), but defects in the tethering of meiotic telomeres to the nuclear envelope, ring chromosomes and chromosome end-to-end fusions have been reported, indicating a putative participation of CDK2 in telomere capping (Viera et al., 2009). In the present study, we demonstrate by electron microscopy the presence of CDK2 at the attachment plates of the synaptonemal complex, suggesting a possible participation of CDK2 in meiotic telomere attachment. Moreover, we found that in $C d k 2^{-/}$spermatocytes a high percentage of telomeres were lying at the nuclear interior in leptotene $(37.72 \%)$, and that this percentage was increased in zygotene and pachytene-like spermatocytes $(48.48 \%$ and $50.52 \%$, respectively). Thus, we speculate that at least two non-mutually exclusive events might lead to the appearance of internal telomeres in the absence of CDK2. The first one implies that some telomeres are unable to attach to the nuclear envelope during leptotene, and therefore remain in the nucleoplasm in further prophase I stages, as described in knockout mouse models for $\mathrm{Smclb}^{-/-}$and Sun1 ${ }^{-1-}$ mice (Adelfalk et al., 2009; Ding et al., 2007). An alternative, and more fascinating possibility, consists in the detachment of telomeres that were initially attached to the nuclear envelope, accounting for the increase in the rate of non-attached telomeres in zygotene and pachytene-like nuclei. It is widely accepted that several chromosome functions can be governed by mechanical stresses transmitted along the chromosome axes (Kleckner et al., 2004). In this regard, physical tensions generated by the ongoing heterologous synapsis in $C d k 2^{-/-}$spermatocytes might spread along chromosomes up to the telomeres, forcing their detachment from the nuclear envelope. This possibility is strongly supported by the presence of SUN1 and membrane vesicles, probably peeled off the INM of the nuclear envelope, at the internal telomeres found in $C d 2^{-/-}$spermatocytes. Indeed, the fact that the number of internal SUN1 foci increased during prophase I progression, as synapsis proceeds, also reinforces this proposal. In this context, it has been shown that SUN1 persists at the axial and lateral elements ends on spread spermatocytes after a harsh preparative technique that disrupts the nuclear envelope (Adelfalk et al., 2009; Ding et al., 2007; Morimoto et al., 2012). Accordingly, SUN1 at detached telomeres in $C d k 2^{-/-}$spermatocytes could bring with them vesicles derived from the nuclear envelope. Based on our data, we propose that either or both events would finally disrupt chromosome movements, and the formation of a bouquet-like arrangement, and explain the meiotic defects previously found in $C d k 2^{-1-}$ spermatocytes (Viera et al., 2009). However, a detailed analysis of meiotic telomere attachment to the nuclear envelope in other mouse models displaying non-homologous synapsis would clarify whether this is a particular phenotype of the $C d k 2^{-/-}$ background, or by contrast if it represents a general consequence of non-homologous synapsis.

In summary, our results point to CDK2 as a key factor governing the assembly, structure and dynamics of the meiotic nuclear envelope in mice. Moreover, CDK2 is essential for the accurate attachment of meiotic telomeres to the nuclear envelope and chromosome movements in prophase I.

\section{MATERIALS AND METHODS \\ Ethics statement}

Animals were kept under SPF barrier conditions at the CNIO animal facility. Animals were handled according to relevant regulatory standards and experiments were approved by the Universidad Autónoma de Madrid Ethics Committee.

\section{Immunofluorescence microscopy}

Adult male C57BL/6 mice (wild-type) and adult $C d k 2^{-1-}$ mice (Ortega et al., 2003) were used for this study. Testes were removed, detunicated and seminiferous tubules then processed for squashing as previously described (Page et al., 1998; Parra et al., 2002). After fixation, the squashed spermatocyte preparations were rinsed three times for $5 \mathrm{~min}$ in PBS, and incubated overnight at $4{ }^{\circ} \mathrm{C}$ with the corresponding primary antibodies diluted in PBS. To detect SYCP3, we employed either a mouse monoclonal antibody (Abcam, ab-12452) or a rabbit polyclonal serum A1 (a gift from Christa Heyting, Wageningen University, Wageningen, The Netherlands) at a 1:100 dilution. To detect SUN1, we used an affinitypurified guinea pig polyclonal antibody raised against a peptide corresponding to amino acids 428-722 of mouse SUN1 (Adelfalk et al., 2009) at a 1:30 dilution. The centrosome was revealed by using a rabbit polyclonal anti-pericentrin antibody generated against the residues 100-600 of mouse pericentrin 1 (Abcam, ab-4448) at a 1:10 dilution. TRF1 was detected with a rabbit polyclonal serum (Alpha Diagnostic International, TRF 12-S), raised against mouse TRF1, at a 1:50 dilution. KASH5 was detected with a rabbit polyclonal antibody generated against the amino acid residues 242-688 of mouse KASH5 (Link et al., 2014), following previous reports (Morimoto et al., 2012), at a 1:30 dilution. The mouse monoclonal $13 \mathrm{~d} 4$ antibody (Alsheimer et al., 1998) was used to reveal LAP-2 at a 1:10 dilution. To detect lamin C2 we employed either a rabbit polyclonal antibody raised against the amino acids 231340 of human lamin A (Santa Cruz Biotechnology, sc-20681) that also recognizes lamin C2 in mouse spermatocytes (Jahn et al., 2010) or an affinity-purified rabbit polyclonal antibody generated against mouse Atype lamins (Jahn et al., 2010) at a 1:10 dilution. Centromeres were revealed with a human anti-centromere antibody (ACA) (Antibodies Incorporated, 15-235) at a 1:50 dilution. A goat anti-RanGAP1 antibody 
(Pichler et al., 2002) was employed to detect RanGAP1 (a gift from Frauke Melchior, Zentrum für Molekulare Biologie der Universität Heidelberg, Heidelberg, Germany) at a 1:10 dilution.

Following three washes in PBS, the slides were incubated for $30 \mathrm{~min}$ at room temperature with secondary antibodies diluted in PBS. The appropriated combinations of the following secondary antibodies were employed for simultaneous double- or triple-immunolabeling: a fluorescein isothiocyanate (FITC)-conjugated donkey anti-mouse IgG antibody (Jackson) at a 1:150 dilution, a Texas-Red-conjugated donkey anti-mouse ML IgG antibody (Jackson) at a 1:150 dilution, a FITCconjugated donkey anti-rabbit ML IgG antibody (Jackson) at a 1:150 dilution, a Texas-Red-conjugated donkey anti-rabbit ML IgG antibody (Jackson) at a 1:150 dilution, a FITC-conjugated goat anti-human IgG antibody (Jackson) at a 1:150 dilution, a FITC-conjugated donkey antiguinea-pig ML IgG antibody (Jackson) at a 1:150 dilution, a Texas-Redconjugated donkey anti-guinea-pig ML IgG antibody (Jackson) at a 1:150 dilution, and a FITC-conjugated donkey anti-goat ML IgG antibody (Jackson) at a 1:40 dilution. Subsequently, slides were rinsed in PBS, and, in double-immunolabeling experiments, counterstained for $3 \mathrm{~min}$ with $5 \mu \mathrm{g} / \mathrm{ml}$ DAPI. After a final rinse in PBS, the slides were mounted in Vectashield (Vector Laboratories) and sealed with nail varnish.

Observations were performed using an Olympus BX61 microscope equipped with a motorized $z$-axis and epifluorescence optics. Image stacks comprising 65 focal planes across the entire spermatocyte nuclei were captured with an Olympus DP71 digital camera controlled by the analySIS software (Soft Imaging System). The complete image stacks were used to analyze the relative distribution of the proteins at a given entire nucleus and for estimating the percentages that are presented at the results section. For a better presentation of the results in the figures, stacks were subsequently processed for obtaining complete $z$-projections (65 focal planes) or partial $z$-projections ( 15 focal planes along the equator of the nucleus) using the public domain software ImageJ (National Institutes of Health). Final images were processed with Adobe Photoshop 7.0 software.

\section{Transmission electron microscopy and immunogold electron microscopy}

Electron microscopy was performed according to standard procedures (Schramm et al., 2011). Testes of wild-type and $C d k 2^{-1}$ mice were fixed for $2 \mathrm{~h}$ at $4{ }^{\circ} \mathrm{C}$ in $2.5 \%$ glutaraldehyde in $50 \mathrm{mM}$ cacodylate buffer. Then, samples were washed in cacodylate buffer and postfixed in $1 \%$ osmium tetroxide for $1 \mathrm{~h}$ at $4^{\circ} \mathrm{C}$. After rinsing several times in water, samples were dehydrated in an ethanol series and embedded in Epon. Ultrathin sections were stained with uranyl acetate and lead citrate. Observations were performed in a Zeiss EM-10 transmission electron microscope operated at $80 \mathrm{kV}$. For immunoelectron microscopy we proceeded as previously described (Schramm et al., 2011). Briefly, 10- $\mu$ m mouse testis cryosections were fixed with acetone for $10 \mathrm{~min}$ at $-20^{\circ} \mathrm{C}$ and air dried. Incubation with a rabbit anti-CDK2 antibody (Abcam, ab-7954), at a 1:50 dilution in PBS, was carried out in a humidified chamber for $4 \mathrm{~h}$ at room temperature. After two rinses in PBS, sections were fixed for $10 \mathrm{~min}$ in $2 \%$ formaldehyde and blocked with $50 \mathrm{mM} \mathrm{NH}_{4} \mathrm{Cl}$. A goat anti-rabbit secondary antibody conjugated to 6-nm-gold particles (Dianova) was incubated overnight at $4^{\circ} \mathrm{C}$ and samples were washed subsequently in PBS. Samples were fixed for $30 \mathrm{~min}$ in $2.5 \%$ glutaraldehyde and postfixed in $2 \%$ osmium tetroxide. After rinsing three times with $\mathrm{H}_{2} \mathrm{O}$, samples were dehydrated in an ethanol series and embedded in Epon. Ultrathin sections were stained with uranyl acetate and lead citrate according to standard procedures.

\section{In vitro kinase assay}

For the in vitro kinase assay, we proceeded as previously reported (Martín et al., 2005). Briefly, testis lysates were prepared with the Precellys 24 tissue homogenizer (Bertin technologies) in immunoprecipitation buffer $(0.5 \%$ Nonidet p-40; $50 \mathrm{mM}$ Tris- $\mathrm{HCl}$ $\mathrm{pH} 7.5 ; 50 \mathrm{mM} \mathrm{NaCl})$ supplemented with the protease inhibitor cocktail (Roche). Lysates were clarified by centrifugation and $500 \mu \mathrm{g}$ were incubated overnight at $4{ }^{\circ} \mathrm{C}$ with $2 \mu \mathrm{g}$ of a rabbit anti-CDK2 antibody (Santa Cruz Biotechnology, sc-163) followed by precipitation with protein-A-sepharose beads for $2 \mathrm{~h}$. Immunoprecipitates were washed three times with $1 \mathrm{ml}$ of immunoprecipitation buffer and three times with kinase buffer (50 mM Hepes $\mathrm{pH} 7.5 ; 50 \mathrm{mM}$ urea; $10 \mathrm{mM}$ $\mathrm{MgCl}_{2} ; 5 \mathrm{mM} \mathrm{MnCl} 2 ; 1 \mathrm{mM}$ DTT). The beads were suspended in $30 \mu \mathrm{l}$ of kinase buffer containing $1 \mu \mathrm{g}$ substrate; either His-tagged Sun1 (amino acids 1-243) or retinoblastoma protein (pRb) (amino acids 773-928, Upstate, 12-439); $30 \mu \mathrm{M}$ ATP and $10 \mu \mathrm{Ci}$ of $\left[\gamma_{-}{ }^{32} \mathrm{P}\right]$ ATP (Perkin Elmer, $6000 \mathrm{Ci} / \mathrm{mmol}$ ). After incubation for $30 \mathrm{~min}$ at $30^{\circ} \mathrm{C}$ with occasional mixing the samples were boiled with SDS/PAGE sample buffer and separated by electrophoresis. Phosphorylated proteins were visualized with a phosphoimager (Storm 820; Molecular Dynamics) after overnight exposure.

\section{Acknowledgements}

We express our sincere thanks to Christa Heyting and Frauke Melchior for providing antibodies. We also thank Miriam García and Isabel Blanco, from the Animal Facility at the CNIO, for their help with mouse maintenance, and Lorena Barreras for her technical assistance.

\section{Competing interests}

The authors declare no competing interests.

\section{Author contributions}

A.V. and J.A.S. conceived and designed the experiments. A.V., M.A., C.E.S., R.B. and J.A.S. performed the experiments. A.V., M.A. R.G., I.B., S.O., C.E.S., D.S., R.B. and J.A.S. analyzed the data. A.V. and J.A.S. wrote the paper and all authors revised the manuscript.

\section{Funding}

This work was supported by the Ministerio de Ciencia e Innovación (Spain) [grant number BFU2009-10987/BCM to A.V.] and the Ministerio de Economía y Competitividad (Spain) [grant number SAF2011-28842-C02-01 from to J.A.S.] M.A. and R.B. are supported by grants of the Priority Program SPP 1384 'Mechanisms of genome haploidization' of the German Science Foundation (DFG) and the Graduate School 'Organogenesis' of the University of Würzburg (Germany). I.B. is supported by a predoctoral fellowship from Ministerio de Economía y Competitividad (Spain). C.E.S. is supported by a grant from the 'La Caixa'/CNIO International Ph.D. Fellowship Program sponsored by 'La Caixa' Foundation (Spain).

\section{Supplementary material}

Supplementary material available online at

http://jcs.biologists.org/lookup/suppl/doi:10.1242/jcs.154922/-/DC1

\section{References}

Adelfalk, C., Janschek, J., Revenkova, E., Blei, C., Liebe, B., Göb, E., Alsheimer, M., Benavente, R., de Boer, E., Novak, I. et al. (2009). Cohesin SMC1beta protects telomeres in meiocytes. J. Cell Biol. 187, 185-199.

Alsheimer, M. (2009). The dance floor of meiosis: evolutionary conservation of nuclear envelope attachment and dynamics of meiotic telomeres. Genome Dyn. 5, 81-93.

Alsheimer, M. and Benavente, R. (1996). Change of karyoskeleton during mammalian spermatogenesis: expression pattern of nuclear lamin $\mathrm{C} 2$ and its regulation. Exp. Cell Res. 228, 181-188.

Alsheimer, M., Fecher, E. and Benavente, R. (1998). Nuclear envelope remodelling during rat spermiogenesis: distribution and expression pattern of LAP2/thymopoietins. J. Cell Sci. 111, 2227-2234.

Alsheimer, M., von Glasenapp, E., Hock, R. and Benavente, R. (1999). Architecture of the nuclear periphery of rat pachytene spermatocytes: distribution of nuclear envelope proteins in relation to synaptonemal complex attachment sites. Mol. Biol. Cell 10, 1235-1245.

Alsheimer, M., von Glasenapp, E., Schnolzer, M., Heid, H. and Benavente, R. (2000). Meiotic lamin C2: the unique amino-terminal hexapeptide GNAEGR is essential for nuclear envelope association. Proc. Natl. Acad. Sci. USA 97, 13120-13125.

Ashley, T., Walpita, D. and de Rooij, D. G. (2001). Localization of two mammalian cyclin dependent kinases during mammalian meiosis. J. Cell Sci. 114, 685-693. Berthet, C., Aleem, E., Coppola, V., Tessarollo, L. and Kaldis, P. (2003). Cdk2 knockout mice are viable. Curr. Biol. 13, 1775-1785.

Crisp, M., Liu, Q., Roux, K., Rattner, J. B., Shanahan, C., Burke, B., Stahl, P. D. and Hodzic, D. (2006). Coupling of the nucleus and cytoplasm: role of the LINC complex. J. Cell Biol. 172, 41-53.

Ding, X., Xu, R., Yu, J., Xu, T., Zhuang, Y. and Han, M. (2007). SUN1 is required for telomere attachment to nuclear envelope and gametogenesis in mice. Dev. Cell 12, 863-872. 
Esponda, P. and Giménez-Martín, G. (1972). The attachment of the synaptonemal complex to the nuclear envelope. An ultrastructural and cytochemical analysis. Chromosoma 38, 405-417.

Franco, S., Alsheimer, M., Herrera, E., Benavente, R. and Blasco, M. A. (2002) Mammalian meiotic telomeres: composition and ultrastructure in telomerasedeficient mice. Eur. J. Cell Biol. 81, 335-340.

Furukawa, K., Inagaki, H. and Hotta, Y. (1994). Identification and cloning of an mRNA coding for a germ cell-specific A-type lamin in mice. Exp. Cell Res. 212, 426-430.

Gerace, L. and Huber, M. D. (2012). Nuclear lamina at the crossroads of the cytoplasm and nucleus. J. Struct. Biol. 177, 24-31.

Hiraoka, Y. and Dernburg, A. F. (2009). The SUN rises on meiotic chromosome dynamics. Dev. Cell 17, 598-605.

Horn, H. F., Kim, D. I., Wright, G. D., Wong, E. S., Stewart, C. L., Burke, B. and Roux, K. J. (2013). A mammalian KASH domain protein coupling meiotic chromosomes to the cytoskeleton. J. Cell Biol. 202, 1023-1039.

Jahn, D., Schramm, S., Benavente, R. and Alsheimer, M. (2010). Dynamic properties of meiosis-specific lamin $\mathrm{C} 2$ and its impact on nuclear envelope integrity. Nucleus 1, 273-283.

Kleckner, N., Zickler, D., Jones, G. H., Dekker, J., Padmore, R., Henle, J. and Hutchinson, J. (2004). A mechanical basis for chromosome function. Proc. Natl. Acad. Sci. USA 101, 12592-12597.

Koszul, R. and Kleckner, N. (2009). Dynamic chromosome movements during meiosis: a way to eliminate unwanted connections? Trends Cell Biol. 19, 716724.

Kracklauer, M. P., Link, J. and Alsheimer, M. (2013). LINCing the nuclear envelope to gametogenesis. Curr. Top. Dev. Biol. 102, 127-157.

Labella, S., Woglar, A., Jantsch, V. and Zetka, M. (2011). Polo kinases establish links between meiotic chromosomes and cytoskeletal forces essential for homolog pairing. Dev. Cell 21, 948-958.

Liebe, B., Alsheimer, M., Höög, C., Benavente, R. and Scherthan, H. (2004). Telomere attachment, meiotic chromosome condensation, pairing, and bouquet stage duration are modified in spermatocytes lacking axial elements. Mol. Biol. Cell 15, 827-837.

Link, J., Jahn, D., Schmitt, J., Göb, E., Baar, J., Ortega, S., Benavente, R. and Alsheimer, M. (2013). The meiotic nuclear lamina regulates chromosome dynamics and promotes efficient homologous recombination in the mouse. PLoS Genet. 9, e1003261.

Link, J., Leubner, M., Schmitt, J., Göb, E., Benavente, R., Jeang, K. T., Xu, R. and Alsheimer, M. (2014). Analysis of meiosis in SUN1 deficient mice reveals a distinct role of SUN2 in mammalian meiotic LINC complex formation and function. PLoS Genet. 10, e1004099.

Mahajan, R., Delphin, C., Guan, T., Gerace, L. and Melchior, F. (1997). A small ubiquitin-related polypeptide involved in targeting RanGAP1 to nuclear pore complex protein RanBP2. Cell 88, 97-107.

Martín, A., Odajima, J., Hunt, S. L., Dubus, P., Ortega, S., Malumbres, M. and Barbacid, M. (2005). Cdk2 is dispensable for cell cycle inhibition and tumor suppression mediated by p27(Kip1) and p21(Cip1). Cancer Cell 7, 591598.

Morimoto, A., Shibuya, H., Zhu, X., Kim, J., Ishiguro, K., Han, M. and Watanabe, Y. (2012). A conserved KASH domain protein associates with telomeres, SUN1, and dynactin during mammalian meiosis. J. Cell Biol. 198, 165-172.
Ortega, S., Prieto, I., Odajima, J., Martín, A., Dubus, P., Sotillo, R., Barbero, J. L., Malumbres, M. and Barbacid, M. (2003). Cyclin-dependent kinase 2 is essential for meiosis but not for mitotic cell division in mice. Nat. Genet. 35, 25-31.

Page, J., Suja, J. A., Santos, J. L. and Rufas, J. S. (1998). Squash procedure for protein immunolocalization in meiotic cells. Chromosome Res. 6, 639-642.

Parra, M. T., Page, J., Yen, T. J., He, D., Valdeolmillos, A., Rufas, J. S. and Suja, J. A. (2002). Expression and behaviour of CENP-E at kinetochores during mouse spermatogenesis. Chromosoma 111, 53-61.

Patel, J. T., Bottrill, A., Prosser, S. L., Jayaraman, S., Straatman, K., Fry, A. M. and Shackleton, S. (2014). Mitotic phosphorylation of SUN1 loosens its connection with the nuclear lamina while the LINC complex remains intact. Nucleus 5, 462-473.

Penkner, A. M., Fridkin, A., Gloggnitzer, J., Baudrimont, A., Machacek, T., Woglar, A., Csaszar, E., Pasierbek, P., Ammerer, G., Gruenbaum, Y. et al. (2009). Meiotic chromosome homology search involves modifications of the nuclear envelope protein Matefin/SUN-1. Cell 139, 920-933.

Pichler, A., Gast, A., Seeler, J. S., Dejean, A. and Melchior, F. (2002). The nucleoporin RanBP2 has SUMO1 E3 ligase activity. Cell 108, 109-120.

Santamaría, D., Barrière, C., Cerqueira, A., Hunt, S., Tardy, C., Newton, K., Cáceres, J. F., Dubus, P., Malumbres, M. and Barbacid, M. (2007). Cdk1 is sufficient to drive the mammalian cell cycle. Nature 448, 811-815.

Sato, A., Isaac, B., Phillips, C. M., Rillo, R., Carlton, P. M., Wynne, D. J., Kasad, R. A. and Dernburg, A. F. (2009). Cytoskeletal forces span the nuclear envelope to coordinate meiotic chromosome pairing and synapsis. Cell 139, 907-919.

Scherthan, H. (2007). Telomere attachment and clustering during meiosis. Cell. Mol. Life Sci. 64, 117-124.

Scherthan, H., Sfeir, A. and de Lange, T. (2011). Rap1-independent telomere attachment and bouquet formation in mammalian meiosis. Chromosoma 120, 151-157.

Schmitt, J., Benavente, R., Hodzic, D., Höög, C., Stewart, C. L. and Alsheimer, M. (2007). Transmembrane protein Sun2 is involved in tethering mammalian meiotic telomeres to the nuclear envelope. Proc. Natl. Acad. Sci. USA 104, 7426-7431.

Schramm, S., Fraune, J., Naumann, R., Hernandez-Hernandez, A., Höög, C., Cooke, H. J., Alsheimer, M. and Benavente, R. (2011). A novel mouse synaptonemal complex protein is essential for loading of central element proteins, recombination, and fertility. PLoS Genet. 7, e1002088.

Smith, A. and Benavente, R. (1992). Identification of a short nuclear lamin protein selectively expressed during meiotic stages of rat spermatogenesis. Differentiation 52, 55-60.

Starr, D. A. and Fridolfsson, H. N. (2010). Interactions between nuclei and the cytoskeleton are mediated by SUN-KASH nuclear-envelope bridges. Annu. Rev. Cell Dev. Biol. 26, 421-444.

Tetsu, O. and McCormick, F. (2003). Proliferation of cancer cells despite CDK2 inhibition. Cancer Cell 3, 233-245.

Tzur, Y. B., Wilson, K. L. and Gruenbaum, Y. (2006). SUN-domain proteins: 'Velcro' that links the nucleoskeleton to the cytoskeleton. Nat. Rev. Mol. Cell Biol. 7, 782-788.

Viera, A., Rufas, J. S., Martínez, I., Barbero, J. L., Ortega, S. and Suja, J. A. (2009). CDK2 is required for proper homologous pairing, recombination and sex-body formation during male mouse meiosis. J. Cell Sci. 122, 2149-2159.

Zickler, D. and Kleckner, N. (1998). The leptotene-zygotene transition of meiosis. Annu. Rev. Genet. 32, 619-697. 